\section{Transplanted liver graft ischemia caused by pediatric ERCP in the prone position}

Endoscopic retrograde cholangiopancreatography (ERCP) for biliary complications after liver transplant is safe and useful in children and adults [1-3]. Here, we report a very rare case of a child in whom liver graft ischemia developed after therapeutic post-transplant ERCP.

A 5-year-old boy, who had received a living donor liver transplant with a reduced left lateral segment graft [4] for Alagille syndrome at 1 year of age, was admitted for cholangitis. Magnetic resonance imaging revealed a bilioenteric anastomotic stricture and hepatolithiasis. ERCP with a short double-balloon enteroscope (EI-530B; Fujifilm, Tokyo, Japan) was performed while the patient was under general anesthesia and in the standard prone position. After the anastomosis had been reached, balloon dilation, stone removal, and the placement of three plastic stents were completed without complications within 2 hours ( Fig. 1). However, 2 hours after ERCP, abdominal pain and acutely elevated liver enzymes (aspartate aminotransferase, $5433 \mathrm{IU} / \mathrm{L}$; alanine aminotransferase, $2161 \mathrm{IU} / \mathrm{L}$; lactate dehydrogenase, $2018 \mathrm{IU} / \mathrm{L}$ ) occurred. Computed tomography revealed a lesion with delayed enhancement and a nonenhanced geographic lesion in the liver graft; the lesions had the appearance of a cylinder whose center axis passed through the top, ventral part of the graft, irrespective of the hepatic segment ( $\bullet$ Fig. 2 ), suggestive of hepatic ischemia with partial infarction. Fortunately, the liver dysfunction decreased with infusion therapy (๑ Fig.3), so that another transplant was avoided. However, 3 months later, the patient's liver volume had slightly decreased (๑ Fig.4).

Hepatic ischemia has been described after prolonged surgery in the prone position [5] but not after ERCP, to the best of our knowledge. In this case, the distribution of a lesion with delayed enhancement on computed tomography suggested that this hemodynamic change had been induced by prolonged, excessive pressure on the liver graft, which protruded ventrally, when it was compressed between the spine and operating table while the patient was in the prone position. Therefore, it is important to note the size and location of a liver graft before a posttransplant patient undergoes ERCP.

\section{Endoscopy_UCTN_Code_CPL_1AK_2AC}

\section{Competing interests: None}
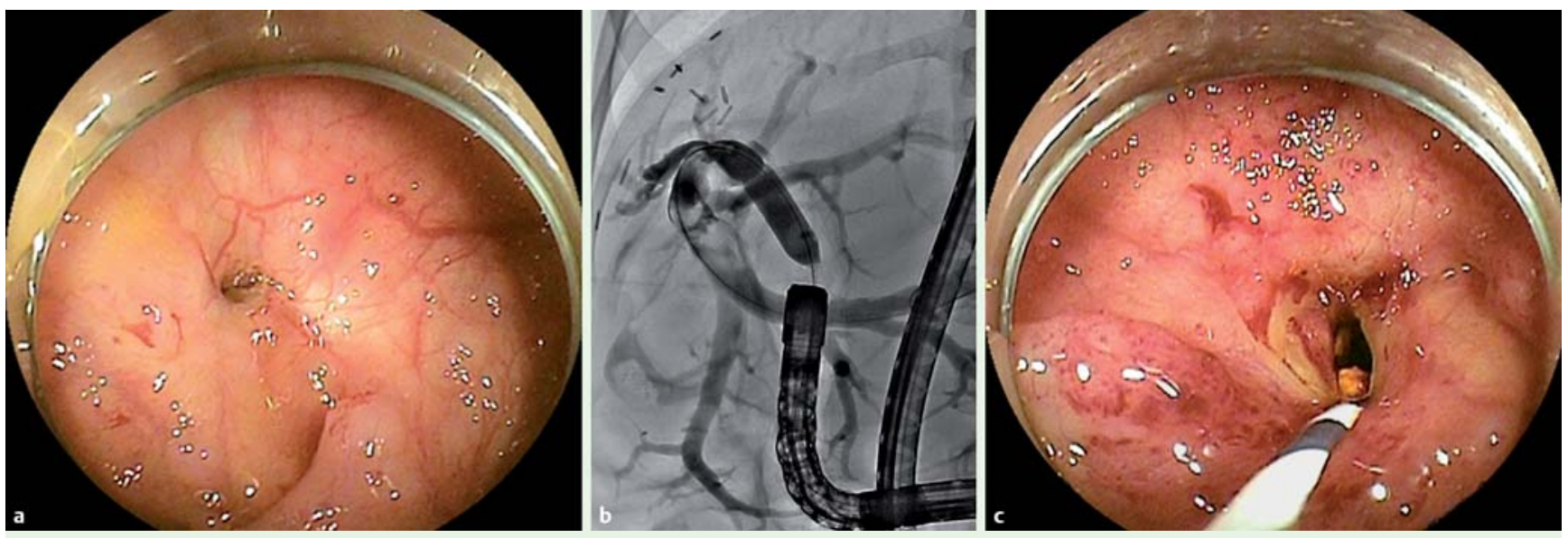

Fig. 1 Therapeutic post-transplant endoscopic retrograde cholangiopancreatography. Endoscopic and cholangiographic views obtained with a short doubleballoon enteroscope showing: $\mathbf{a}$ a bilioenteric anastomotic stricture; $\mathbf{b}$ a balloon dilation (8-mm diameter) of the anastomotic stricture; and $\mathbf{c}$ a widely patulous anastomosis.
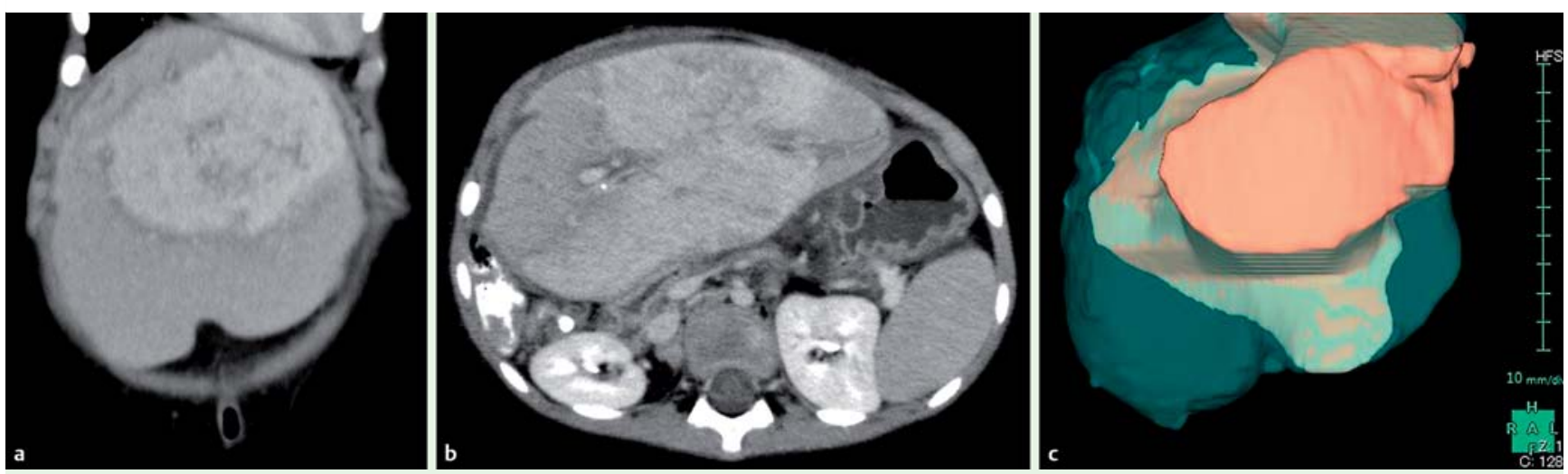

Fig. 2 Computed tomography at 1 day after endoscopic retrograde cholangiopancreatography. a Coronal image. b Axial image. c Three-dimensional image of a lesion with delayed enhancement. 

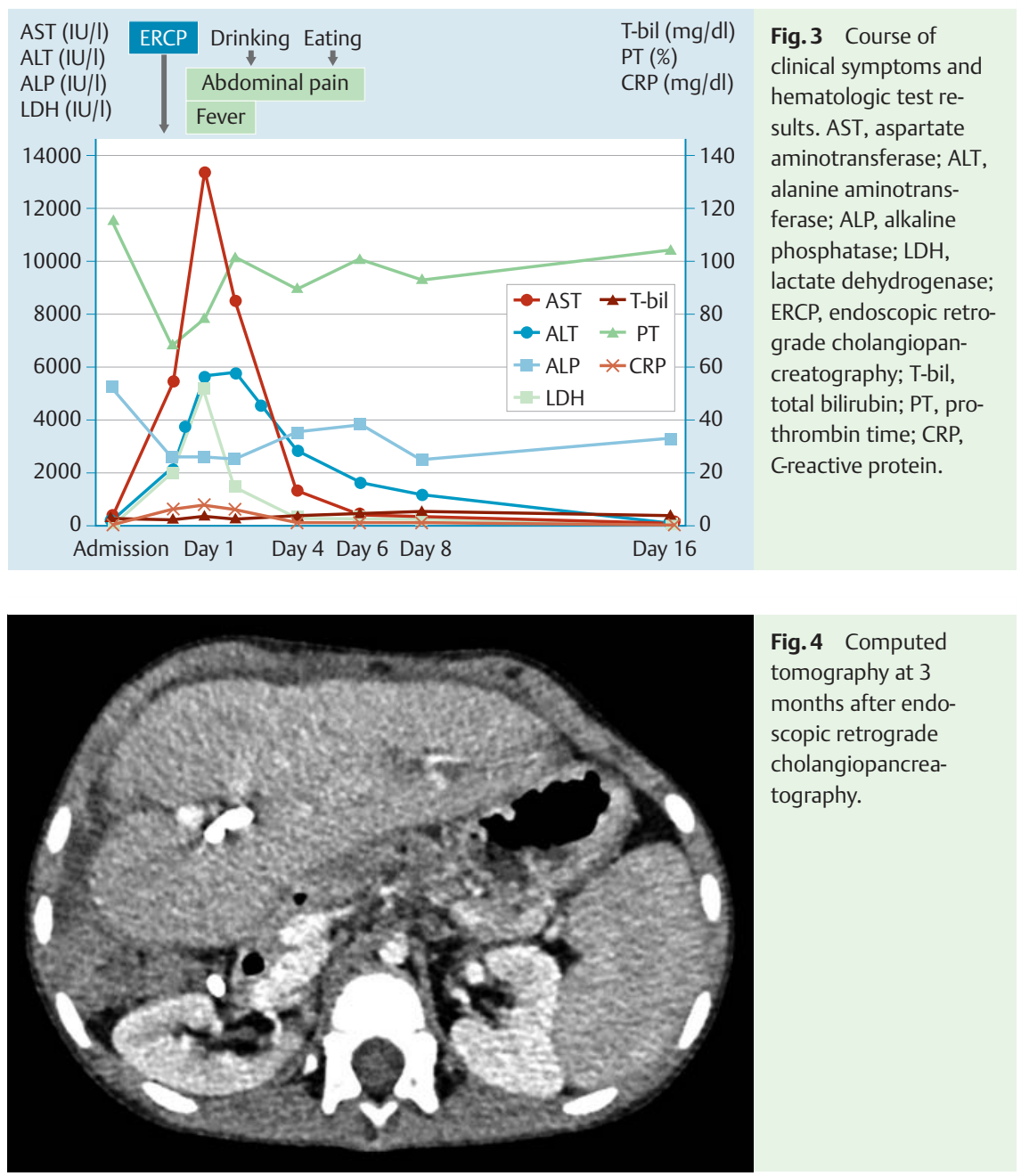

Koichiro Tsutsumi ${ }^{1}$, Hironari Kato ${ }^{1}$, Hiroyuki Okada', Takahito Yagi ${ }^{2}$, Kazuhide Yamamoto ${ }^{1}$

${ }^{1}$ Department of Gastroenterology and Hepatology, Okayama University Graduate School of Medicine, Dentistry, and Pharmaceutical Sciences, Okayama, Japan

${ }^{2}$ Department of Gastroenterological Surgery, Transplant, and Surgical Oncology, Okayama University Graduate School of Medicine, Dentistry, and Pharmaceutical Sciences, Okayama, Japan

\section{References}

1 Otto AK, Neal MD, Mazariegos GV et al. Endoscopic retrograde cholangiopancreatography is safe and effective for the diagnosis and treatment of pancreaticobiliary disease following abdominal organ transplant in children. Pediatr Transplant 2012; 16 : 829-834

2 Sanada Y, Mizuta K, Yano T et al. Double-balloon enteroscopy for bilioenteric anastomotic stricture after pediatric living donor liver transplantation. Transpl Int 2011; 24: $85-90$

3 Arain MA, Attam R, Freeman ML. Advances in endoscopic management of biliary tract complications after liver transplantation. Liver Transpl 2013; 19: 482-498

4 Enne M, Pacheco-Moreira L, Balbi E et al. Liver transplantation with monosegments. Technical aspects and outcome: a meta-analysis. Liver Transpl 2005; 11: 564-569

5 Edgcombe H, Carter K, Yarrow S. Anaesthesia in the prone position. Br J Anaesth 2008; 100: $165-183$

Bibliography

Dol http://dx.doi.org/

10.1055/s-0034-1390732

Endoscopy 2014; 46: E594-E595

(c) Georg Thieme Verlag KG

Stuttgart · New York

ISSN 0013-726X

Corresponding author

Koichiro Tsutsumi, MD, PhD

Department of Gastroenterology and Hepatology Okayama University Graduate School of Medicine, Dentistry, and Pharmaceutical Sciences

2-5-1 Shikata-cho, Kita-ku, Okayama City Okayama 700-8558

Japan

Fax: +81-86-225-5991

tsutsumi@cc.okayama-u.ac.jp 